\title{
INCORPORACIÓN DE LA LITERATURA COSTARRICENSE AL AULA DE ESPAÑOL COMO SEGUNDA LENGUA: UNA PROPUESTA DIDÁCTICA A PARTIR DEL TEXTO BANANOS $Y$ HOMBRES, DE CARMEN LYRA
}

\author{
Incorporation of the Costa Rican literature to the spanish classroom as a second language: \\ a teaching proposal from the text "Bananos y Hombres", by Carmen Lyra
}

\section{Patricia Guillén Solano*}

\begin{abstract}
RESUMEN
Este artículo presenta una propuesta didáctica para la incorporación de la literatura costarricense en el aula de español como segunda lengua. Para ello, se parte del texto Estefanía, uno de los relatos de la obra Bananos y hombres de Carmen Lyra, y se aplica un modelo didáctico específico para su estudio en el aula, mediante el cual se exploran los conceptos de sujeto cultural y marginación.

Palabras clave: literatura costarricense; español como segunda lengua; modelo didáctico; sujeto cultural; marginación
\end{abstract}

\begin{abstract}
This article presents a didactic model for the incorporation of the Costa Rican literature in the Spanish as a second language classroom. This model is applied to the text Estefania, one of the stories contained in Bananos y hombres of Carmen Lyra, and through it, the concepts of marginalization and cultural subject are studied.

Key Words: Costa Rican literature; Spanish as a second language; didactic model; cultural subject; marginalization
\end{abstract}

Universidad de Costa Rica, Departamento de Lingüística, Costa Rica.

Correo electrónico: patricia.guillen@ucr.ac.cr

Recepción: 05/05/14. Aceptación: 13/05/14. 


\section{Introducción}

De acuerdo con Amoretti (2007), en relación con la literatura, las necesidades e intereses de los estudiantes de español como segunda lengua pueden resumirse en dos: reforzar su aprendizaje de la lengua e indagar sobre la cultura que la produce. En el caso de la presente propuesta, se parte de dicha premisa pero enfatizando la segunda necesidad: se trata de estudiantes anglohablantes que se encuentran en Costa Rica en un programa de inmersión y que, debido a su nivel avanzado, toman cursos especializados, entre ellos, un curso de literatura costarricense.

Siguiendo a la autora mencionada, la literatura nos permite acercarnos de forma más contextualizada a la cultura meta. Aun cuando la imagen de esta cultura está trabajada estéticamente y este proceso la transforma, el acercamiento es igualmente válido, pues en el aula de segundas lenguas la literatura puede promover el estudio y discusión de visiones y circunstancias que rodean a los grupos sociales. A su vez, también se convierte en un medio para evidenciar el potencial creativo que tiene la lengua y estudiar patrones de interacción ya contextualizados, lo cual enriquece y aporta más autenticidad a las dinámicas de clase. Dichos patrones de interacción permiten la creación de mundos posibles que, si bien pueden no ser reales, responden a manifestaciones culturales particulares en determinadas épocas. Para Amoretti "El hecho de que el texto literario esté escrito por un intelectual o por escritores que se ubican en un determinado grupo social, no excluye una visión de conjunto de la sociedad y la representación de diversos sectores sociales" (2007: 7).

Con la implementación de las estrategias metodológicas necesarias, la literatura puede ser un recurso para ejercitar las cuatros destrezas básicas (lectura, escucha, habla y escritura), propiciando discusiones sobre los posibles significados del texto a partir de las situaciones que estos plantean, aprovechando, de acuerdo con Amoretti (2007:9) "su relevancia humana y universal".

Así, con base en la riqueza del texto literario y en sus posibilidades de estudio, este artículo plantea una propuesta didáctica para estudiantes de español como segunda lengua de nivel avanzado, aprovechando para ello el interés de estos en probar su dominio de la lengua en una dimensión más compleja. Dicha propuesta corresponde a uno de los doce modelos didácticos planteados por Amoretti (2007), enmarcados dentro del enfoque comunicativo ${ }^{1}$. Para su aplicación, partimos de la premisa metodológica de que el conocimiento compartido, al que la autora también denomina "capital cultural propio" y que es adquirido en un espacio social determinado, permitirá discutir y presentar aspectos propios de la cultura "ajena" o cultura meta inmersos en el texto literario. Para llevar a cabo esta tarea, hemos elegido el texto Estefanía, uno de los relatos de la obra Bananos y hombres de Carmen Lyra (1931).

\section{Selección del texto y del modelo didáctico}

Amoretti (2007) afirma que, en el caso de la enseñanza de segundas lenguas, el profesor de literatura debe tener un conocimiento aceptable de la literatura como discurso social y estético y manejar un mínimo de estrategias para su enseñanza en el aula. Partiendo de dicha premisa, presentamos a continuación una contextualización del texto seleccionado y la explicación del modelo didáctico con el cual se trabajará en el aula.

\subsection{El relato "Estefanía"}

La propuesta se centra en el análisis del relato Estefanía, el cual forma parte de una serie de textos titulada Bananos y hombres, que se publicó en Repertorio Americano en 1931 y en el periódico Trabajo en 1934 (Araya y Ovares, 1985). La obra completa consta de cuatro relatos: "Estefanía", "Nochebuena", "Niños" y 
"Río arriba". Tal como lo indicamos, hemos seleccionado el primero de ellos para nuestra propuesta. En este caso, el motivo de dicha selección está basado en un contenido del curso de literatura costarricense que deben abarcar los estudiantes como parte de su programa de inmersión de un mes en la Universidad de Costa Rica. Dicho contenido está esbozado como "La generación del 40". De acuerdo con Araya y Ovares (1985), la obra de Carmen Lyra Bananos y hombres pertenece a un período de transición entre la generación del novecientos, donde destacan Joaquín García Monge y Carlos Gagini como pioneros de la narrativa realista y la llamada "Generación del 40", que consolida la temática agraria y social. De hecho, siguiendo a las autoras, Carmen Lyra es considerada guía intelectual de dicha generación, en la que se incluyen autores como Adolfo Herrera García, Carlos Luis Fallas y Fabián Dobles, y dentro de la cual Bananos y hombres es considerada "la primera elaboración literaria del mundo bananero en Costa Rica" (Araya y Ovares, 1985: 104).

Lyra sitúa los problemas concretos y específicos de los bananeros en un contexto costarricense, donde se manifiesta la subordinación respecto de los hacendados. En el plano internacional, se destaca la dependencia en relación con las corporaciones extranjeras, exponiendo en detalle el comienzo del capitalismo monopolista en Costa Rica y situando a los personajes de Bananos y hombres en la primera época de funcionamiento del enclave bananero, entre 1880 y 1934 . Implica la presencia de la United Fruit Company en el Atlántico y la importación de la mano de obra de los negros antillanos. Hacia 1934 la compañía se traslada al Pacífico, dejando en la zona atlántica una población desarraigada, excluida de la actividad económica del país y una región de tierras agotadas (Araya y Ovares 1985:105).

Para las autoras, en los relatos de Bananos $y$ hombres el punto de vista narrativo opta por el paradigma naturalista, pero lo transforma al cuestionar su carga ideológica y le confiere una modalidad específica de acuerdo con una óptica histórica más amplia, sin dejar de lado varios rasgos de dicho paradigma, por ejemplo: la predilección por el carácter utilitario y militante de la literatura, el punto de vista narrativo que se afianza en la percepción sensorial con la finalidad de expresar los rasgos característicos de una esfera social así como las miradas constantes de la voz narradora sobre la exterioridad de los personajes, incluyendo la descripción y evidencia de síntomas de enfermedades que afectan a las mujeres trabajadoras de la región, consideradas como un grupo con problemas comunes que define un sector importante de la nacionalidad.

Hay que señalar también que los estratos populares desempeñan un papel protagónico, en este caso se destaca el proletariado agrícola. En el caso del relato "Estefanía" se observa la degradación del personaje femenino, cuyo personaje es no solo individual, sino símbolo del destino de un sector social. Así, Estefanía es el prototipo de la mujer de la zona bananera, sin apellidos y marginada.

No obstante las características mencionadas, para Araya y Ovares (1985) ciertos rasgos de los relatos intentan una superación del paradigma naturalista, en tanto corresponden a un esfuerzo por superar los límites de la crítica naturalista. Los relatos otorgan prioridad a las motivaciones políticas y sociales sobre la causalidad propia de las ciencias naturales. Desaparece el determinismo racial y la idea de que el medio geográfico es el elemento que degrada a los personajes, pues el momento histórico es la razón fundamental de los conflictos. Los problemas particulares se hacen generales y se engloban en una situación más amplia. Para abordar las situaciones en las que se inserta el personaje, Lyra utiliza la técnica de la comparación y el contraste: Describe el estado de las mujeres de la clase popular y la contrasta con los privilegios que disfruta la clase adinerada, poniendo en evidencia la explotación a la cual está sometida Estefanía.

Secundamos la opinión de Araya y Ovares (1985) en cuanto a que el tema del machismo está también contemplado como parte de la opresión organizada por el sistema. El 
machismo es entendido como una faceta de la explotación general, como se evidencia en el siguiente fragmento:

Creo que en Santa Cruz, el juez que más tarde llegó a ser un honorable magistrado de la Corte de Justicia, le hizo un chiquillo cuando ella apenas entraba en la adolescencia. Por supuesto que después el estimable caballero ni se acordaba de la insignificante aventura. Ella dejó al hijo en la primera casa propicia y comenzó a rodar. Luego otro, ella ni recordaba bien el nombre, la dejó embarazada y siguió rodando, rodando. (Lyra, 1931: 372)

En conclusión, nos decantamos por esta obra en tanto muestra un carácter fundacional en relación con la literatura de tema bananero en Costa Rica y evidencia la opresión de la mujer mostrando las condiciones de su existencia.

Resulta también de importancia para nuestra propuesta didáctica que la producción de Carmen Lyra se enmarque dentro de lo que Amoretti define como literatura femenina:

\begin{abstract}
Conjunto de textos considerados en función de su sujeto empírico; en otras palabras, la literatura femenina reúne el corpus de textos literarios escritos por mujeres y que se distingue, a su vez, de la literatura feminista, la cual se refiere a la postura militante e ideológica que uno puede encontrar en determinado texto." (2007: 113).
\end{abstract}

El motivo por el cual resulta importante es que, de acuerdo con la autora, se distinguen varios rasgos de la literatura femenina, de los cuales hemos seleccionado tres para pedirles a las estudiantes (valga resaltar que el grupo para el cual se dirige la propuesta está constituido por seis mujeres) que los identifiquen en el texto, a saber:

- La insignificancia del personaje masculino en relación con el femenino.

- La preferencia por el tiempo circular o cíclico, pero presentado en sentido cualitativo y no cuantitativo, como se observa en la escritura masculina.

- La preferencia por el enfoque del tipo close up en lugar del gran angular.

Es necesario aclarar que la autora señala que estas y otras características tienen como función legitimar una diferencia entre la escritura femenina y la escritura masculina, no absolutizar un nuevo canon.

\subsection{El modelo didáctico}

En nuestra propuesta también rescatamos la especificidad de la literatura femenina en tanto la producción de mujeres está inserta en la problemática de los discursos marginales $\mathrm{y}$, precisamente, tomamos como eje el esquema de la marginación para abordar el texto. Dentro de esta marginación, de acuerdo con Amoretti (2007), una de las mayores dificultades de la voz femenina es la dificultad de decir yo, dentro de un modelo patriarcal que relaciona lo femenino con la maternidad y la sumisión, como se evidencia en el apartado anterior.

Para plantear las actividades de clase seleccionamos el modelo didáctico basado en esquemas. En este caso, el modelo, patrón o esquema cultural consiste en una representación abstracta de un concepto genérico relativo a un objeto, un evento o situación. La teoría del esquema destaca el papel que juega la experiencia o el conocimiento previo en la comprensión. Desde ese punto de vista, el texto ofrece instrucciones para que el lector pueda construir un significado desde su propia estructura cognoscitiva, la cual ha sido adquirida por su experiencia previa. Las estructuras previamente adquiridas reciben el nombre esquemata y hacen que la interpretación se vea influida por la historia personal, intereses, ideas preconcebidas y antecedentes culturales del lector. Se tratará en el proceso de hacer calzar el significado del mensaje en el esquema que uno tiene en la mente (Amoretti, 2007: 138).

En la lectura por esquemas se da una interacción entre dos formas de procesar la información: el proceso ascendente de tipo inductivo, que va de los detalles a la imagen total, o el proceso descendente de tipo deductivo, que va desde la imagen global hacia el detalle. Este último es el modelo que hemos seleccionado.

Así, este tipo de actividad de lectura consiste en trabajar a partir de un esquema 
previo y sus asociaciones, para interpretar luego el texto en función del esquema, por lo cual tiene la ventaja de facilitar la captación de la imagen total del texto por parte del estudiante y orientar las operaciones inferenciales. Sin embargo, hemos de reconocer que, tal como lo indica la autora, coarta su creatividad personal en la lectura y limita la expresión personalizada de la lectura como experiencia productiva. Pese a esta desventaja, creemos que resulta adecuada para iniciar un acercamiento general a la literatura costarricense en el aula de español como segunda lengua.

Si bien no debemos sobregeneralizar patrones culturales, el sentido solo es posible captarlo gracias a un saber compartido, por lo cual la literatura llega a ser un insumo en el estudio de la cultura si partimos de que debe ser analizada de forma que se hagan explícitos los textos culturales insertos en ella (Amoretti, 2007).

Concluimos entonces que el significado que damos a los textos refleja en gran medida un conocimiento compartido. En este estudio dicho conocimiento compartido se explora a partir del término marginalidad y de la noción de sujeto cultural de Cross:

[...] una instancia que integra a todos los individuos de la misma colectividad: en efecto, su función objetiva es integrar a todos los individuos en un mismo conjunto al tiempo que los remite a sus respectivas posiciones de clase, en la medida en que, como ya he dicho, cada una de esas clases sociales se apropia de ese bien colectivo de diversas maneras (2003: 12).

El sujeto cultural implica, de esta forma:

- $\quad$ Una instancia de discurso ocupada por un yo.

- La emergencia y el funcionamiento de una subjetividad

- Un sujeto colectivo

- $\quad$ Un proceso de sumisión ideológica.

Para abordar el concepto de marginación, tomamos como base la definición de Bustos (2011: 176): “La marginación es un fenómeno estructural múltiple que valora dimensiones, formas e intensidades de exclusión en el proceso de desarrollo y en el disfrute de sus beneficios".

\section{Aplicación del modelo}

\subsection{Objetivos propuestos y nivel de competencia esperado}

La utilización del modelo didáctico basado en esquemas aplicado al texto "Estefanía" tiene los siguientes objetivos:

1. Identificar los rasgos que caracterizan a Estefanía como un personaje objeto de marginación.

2. Discutir la caracterización del personaje a partir del concepto de marginación.

La unidad está planteada para el nivel C1, en el cual, de acuerdo con el MCER (2002:31) los estudiantes son capaces de:

- $\quad$ Expresarse con fluidez y espontaneidad sin tener que buscar de forma muy evidente las expresiones adecuadas.

- Utilizar el lenguaje con flexibilidad y eficacia para fines sociales y profesionales.

- $\quad$ Formular ideas y opiniones con precisión y relacionar intervenciones hábilmente con la de otros hablantes.

- $\quad$ Presentar descripciones claras y detalladas sobre temas complejos que incluyen otros temas, desarrollando ideas concretas y terminando con una conclusión apropiada.

\subsection{Actividades}

Para esta lección, se parte de que en la clase anterior el profesor asignó un proyecto corto de investigación en parejas: cada pareja debió investigar sobre los aportes de Carmen Lyra en tres campos específicos: folclore popular, ensayo y narrativa de tono social y campo educativo. La clase inicia con la exposición de los resultados.

Posteriormente, se les solicita a las estudiantes que salgan de la clase durante un minuto. Mientras tanto, el profesor coloca 
un sobre con el nombre de cada una de las estudiantes en sus respectivos asientos. Pasado el minuto, les solicita que entren a la clase y abran el sobre. Ahí encontrarán cuatro fotografías, iguales para cada estudiante, que representan los conceptos de dinero, comida, salud, familia). Se les pide que ordenen esas fotografías de acuerdo con la categoría +importante-importante en la vida. Después de un minuto, cada estudiante pasa a la pizarra y les muestra a sus compañeras el orden de sus fotografías. También explica por qué eligió este orden.

- Una vez terminada la actividad, el profesor coloca en la pizarra el siguiente cartel:

\begin{abstract}
Pongo primero bananos que hombres porque en las fincas de banano, la fruta ocupa el primer lugar, o más bien el único. En realidad el hombre es una entidad que en esas regiones tiene un valor mínimo y no está en el segundo puesto, sino que en la punta de la cola de los valores que allí se cuentan (Lyra, 1931: 371)
\end{abstract}

Se les pregunta a las estudiantes qué diferencias encuentran entre esa afirmación y el orden que eligieron en la fotografía ( $i a$ qué le dieron prioridad?).

El profesor explica que el fragmento escrito en el cartel pertenece al texto Bananos $y$ hombres, el cual se sitúa en el contexto de la explotación bananera en Costa Rica durante finales del siglo XIX y principios del siglo XX y que fue publicado en 1931.

Una razón fundamental para la elección de este texto es su extensión. El hecho de que sea corto, unido al nivel de competencia que tienen los estudiantes, permite efectuar su lectura en el aula, para lo cual se divide la clase en dos grupos. Antes de asignar la tarea por realizar, el profesor indica que la actividad que sigue está basada en la vida de Estefanía R: "una de las tantas mujeres que han pasado por las fincas de banano en Costa Rica". A cada grupo se le asigna un fragmento del texto Estefanía, el cual procederán a leer en silencio. Juntos, harán una lista de vocabulario desconocido. Posteriormente, con la participación de todo el grupo, se aclarará el vocabulario correspondiente.
Una vez aclaradas las dudas, se divide la clase en dos grupos y se le solicita a cada grupo que identifique los rasgos presentes en el texto que caracterizan a Estefanía como un personaje objeto de marginación y elabore un dibujo colectivo con base en la siguiente afirmación: "Estefanía representa la marginación." El profesor aporta material para la actividad: marcadores, cartulina, revistas, tijeras y goma, en caso de que deseen extraer imágenes o fotos que les sean útiles para su presentación.

Seguidamente, el grupo selecciona a un miembro para que exponga el dibujo y lo explique.

La información obtenida por cada grupo se expone y se compara.

Como actividad de cierre, el profesor retoma la información esbozada en la sección 1.1. del presente artículo y la presenta a la clase. Por último, les solicita a las estudiantes que, de forma individual, identifiquen en el relato "Estefanía" las tres características de la literatura femenina vistas en clase y las apoyen con ejemplos concretos en el texto. La clase termina con la discusión de los resultados.

\section{Conclusión}

Este artículo presenta una propuesta didáctica para la incorporación de la literatura costarricense en el aula de español como segunda lengua. Para ello, toma como eje el texto Estefanía, uno de los relatos de la obra Bananos y hombres de Carmen Lyra. A partir del modelo didáctico basado en esquemas, se desarrolla una serie de actividades de clase para el estudio del texto mencionado, mediante las cuales se pretenden explorar los conceptos de sujeto cultural y marginación.

Apoyamos el criterio de Amoretti (2007) en cuanto a que el estudio de la literatura en el aula puede promover la discusión e intercambio de visiones y circunstancias que rodean a los grupos sociales $\mathrm{y}$, por tanto, instamos a la creación de actividades específicas utilizando modelos didácticos que propicien la identificación de patrones culturales, para cuya 
discusión y análisis se parta de una integración de destrezas que evidencie el manejo de la lengua en sus diferentes dimensiones.

Por último, esperamos que las actividades propuestas puedan ejecutarse en el aula de español como segunda lengua y que generen nuevas propuestas.

\section{Notas}

1. Como premisas fundamentales, el enfoque comunicativo plantea hacer de la competencia comunicativa la meta de la enseñanza de lenguas y desarrollar procedimientos para la enseñanza de las cuatro destrezas. La lengua es el vehículo para la expresión de significado funcional, por lo cual la competencia comunicativa implica el uso de la lengua para:

Obtener cosas (función instrumental)

Controlar el comportamiento de otros (función regulatoria)

Propiciar interacciones con otros (función interaccional)

Expresar sentimientos (función personal)

Aprender y descubrir (función heurística)

Crear mundos imaginarios (función imaginativa)

Comunicar información (función representacional) (Richards, Jack.C y Theodore S. Rodgers, 2001)
La autora destaca que este enfoque permite, en el caso del análisis de textos literarios, que el significado se enriquezca con el diálogo que se establece entre literatura-pensamiento-cultura e identidad.

\section{Bibliografía}

Amoretti-Hurtado, María. (2007). Didáctica de la literatura en la enseñanza de segundas lenguas. San José: Editorial UCR.

Araya-Solano, Seidy., Ovares-Ramírez, Flora. (1985). "Las manifestaciones intertextuales de Bananos y hombres de Carmen Lyra". Káñina. Revista de Artes y Letras de la Universidad de Costa Rica. 9 (2): 103-108.

Bustos, Alfredo. (2011). "Realidad, datos y espacio". Revista internacional de estadística. 2 (1): 169-186

Cros, Edmond. (2003). El sujeto cultural. Sociocrítica y psicoanálisis. Medellín: Fondo Editorial Universidad, EAFIT. Segunda edición corregida y aumentada.

Lyra, Carmen. (1931). Bananos y hombres. Repertorio Americano: 371-375.

Richards, Jack C. Rodgers, Theodore S.. (2001). Approaches and methods in language teaching. Cambridge: Cambridge University Press. 